\title{
UTILITY OF PRACTICAL SCORE IN EARLY DIAGNOSIS OF ACUTE APPENDICITIS
}

Ashok Surybhanji Gajbhiye, Malikchand M. Meshram, Amrish P. Kathod

1. Assistant Professor. Department of General Surgery, IGGMC, Nagpur.

2. Assistant Professor. Department of General Surgery, IGGMC, Nagpur.

3. Assistant Professor. Department of General Surgery, IGGMC, Nagpur.

\section{CORRESPONDING AUTHOR:}

Ashok Surybhanji Gajbhiye,

Plot no- 54, Kalpatarunagar,

Manewada, Nagpur- 34.

E-mail: gsashok1972@gmail.com

ABSTRACT: AIM: Acute appendicitis should be diagnosed accurately and precisely, so that appendectomy is neither at the cost of morbidity nor at the cost of a normal appendix. PATIENTS AND METHODS: - The prospective validation of scoring system was conducted in the department of surgery, IGGMC, Nagpur. During the period from May 2002 to Jan 2012 total 683 patients with preoperative diagnosis of acute appendicitis were included in the study. We have developed a scoring system by using bedside six parameters and their scores which were proved to be significant in accurate diagnosis of acute appendicitis. RESULTS:-The number of histopathologically proved acute appendicitis patients in our study is 596. Out of these, 18 patients had perforated appendix. The overall incidence of incorrect diagnosis of acute appendicitis was $12.74 \%$. Positive predictive value is $70.06 \%$. CONCLUSION:-The devised scoring system help to reduce the negative appendectomy rate and it can be good assistance in suspected cases of acute appendicitis for making an accurate diagnosis. If the present scoring system is followed the negative appendicectomy rate would be $4.69 \%$. However, further randomized control trial is needed to investigate the clinical benefit of practical scoring in acute appendicitis.

INTRODUCTION: Acute appendicitis is one of the most common abdominal condition by which a surgeon can come across. No measure is known to prevent the occurrence of the disease. Aetiological factors still remain vague and indefinite. Absolute diagnosis of acute appendicitis is only possible at operation and histopathologic examination of specimen. Appendicectomy continues to be the treatment of choice since 1887 when Claudius Amyand did the first appendicectomy. Laparoscopy is a technique for both diagnosis and treatment of appendicitis. Laparoscopic appendicectomy does not give cost benefit and the operative time may be longer but cosmesis, postoperative pain, time to return to activity and wound infection is decreased.

The appendix is found to be normal in 8 to $21 \%$ of explorations and $15 \%$ cases have perforated appendix which increases the morbidity of the patient which is due to delay in diagnosis[1 ].In order to reduce the negative appendicectomy rates various scoring system have been developed for supporting the diagnosis of acute appendicitis[2,3,4,5,6,7,8]. Alvarado Score was developed in 1986 (Score more than 7 indicate the need for surgery). This score included left shift of neutrophil maturation which is not routinely available in many laboratories. Therefore, Kalan et al [5] omitted this parameter and produced a Modified Alvarado Score (score 7 to 9 are regarded as probable acute appendicitis), Teichers Score in 1983 (score more than +2 need immediate operation), Christian and Christian in 1992( If 4 or 5 criteria were 
present on admission appendicectomy was carried out), Bayesion( consist of 19 attributes which are self explanatory and end score more than +12 is cutoff level for recommendation for laparotomy), Lindberg in 1988(Threshold score $\geq 0.45$ need operation),

Ohmann score [7] allocated 8 point in his score (score $\geq 12$ recommended immediate operation). Fenyos score [8] sensitivity was 0.73 and specificity was $0.87 \%$.

Accuracy of intraoperative diagnosis of appendicitis showed that correct intraoperative diagnosis was made in $84 \%$ of removed appendices and $29 \%$ of appendices thought to be macroscopically normal and removed were found to be appendicitis after histologic examination. They advocate removal of a normal looking appendix in the absence of other explanatory pathology $[9,10]$.

We have developed our practical scoring prospectively to evaluate six significant parameters which can be evaluated at the bedside of patient and by using standard statistical methods; their application in accurate diagnosis of acute appendicitis was done.

PATIENTS AND METHOD: The prospective validation of the scoring system was conducted in department of surgery, IGGMC, Nagpur from May 2002 to Jan 2012. During the study period, 683 patients with preoperative diagnosis of acute appendicitis were included in the study. Patients were seen by the consultants. Infants, appendicular lump and generalized peritonitis were excluded from the study. The weightage in scores was also given to x-ray findings, USG findings, CT findings and to other clinical parameters but discarded them scientifically by comparing these scores with the outcome of these parameters.

Finally we were used six parameters and their scores which were proved to be significant in the accurate diagnosis of acute appendicitis after compilation of collected data from 683 patients. The data were subjected to univariate and multivariate analysis. The coefficient value of the factors is multiplied by minus 10 , it gave rough scores for this significant six factors. Depending on this approximate score a tentative scoring is developed after rounding of score to nearest figure and dividing by 3 . Thus the tentative scores for the following significant six factors are shown in table -1 to diagnosed acute appendicitis preoperatively.

Table - 1 Showing variables and their score.

\begin{tabular}{|l|l|l|}
\hline Sr. no. & Variable & Score \\
\hline 1 & Pain shifting to right iliac fossa & 6 \\
\hline 2 & Rebound tenderness & 5 \\
\hline 3 & Nausea / vomiting & 4 \\
\hline 4 & PR tenderness on right wall of rectum & 3 \\
\hline 5 & Duration of pain $\leq 24 \mathrm{hrs}$ & 0.2 \\
\hline 6 & Pulse $>90 / \mathrm{m}$ & -0.1 \\
\hline
\end{tabular}

A total score for each individual patient was obtained by adding the corresponding statistical weights for the above six factors present in the patients. The Sensitivity, Specificity, Predictive accuracy, Likelihood ratio and Cohen Kappa statistics were calculated at various cut offs of the total score by using computer software XLSTAT (Ver.8, 2012).The best cut offs for the total score was obtained by plotting the ROC Curve. The area under the ROC Curve was used to assess the predictive accuracy of the scoring system.

All patients were informed about the scoring system. Clinician's decision was considered as final for individual patient therapy. The result of scoring system was not accepted as decisive 
for choice of therapy but was regarded only as diagnostic aid. When no detectable pathology was found, appendicectomy was carried out and specimen was sent for histopathological examination.

RESULTS: The present study consists of 683 patients of acute appendicitis. The number of histopathologically proved acute appendicitis patients in our study is 596 and 87 patients were histopathologically negative for acute appendicitis. Out of 596, 18 patients had perforated appendix. The overall incidence of incorrect diagnosis of acute appendicitis is $12.74 \%$. Out of 87 patients, 79 patients had no detectable pathology and 8 patients had some other pathology than acute appendicitis. Out of these 8 patients, 5 patients [Meckel's diverticulitis $(n=3)$, Ruptured ectopic pregnancy $(n=2)$ ] required operative management and for 3 patients [Mesentric adenitis $(n=1)$, T.0 mass $(n=2)]$ non operative treatment were needed.

Most of the patients with positive appendicectomy were in 11to30 year's age group i.e. in the younger age group. The incidence is very low in the age group of below 10 years and above 40 years. 362(60.73\%) positive appendicectomies patients were in the males while female comprises 53(60.92\%) of the negative appendicectomy group. The male: female ratio in the patients with positive appendicectomy is $1.5: 1$. The total negative appendicectomies is in women between the age group of $11-30$ years was $60.92 \%$. This age group includes the peak child bearing age of women (20-30 years).

Retrocaecal position of appendix was the commonest and it was present in $73.50 \%$ and postileal position was in $9.80 \%$ of the patients. The mean hospital stay was around 5.18 days of all the operated patients with diagnosis of acute appendicitis. The mean hospital stay in patients with histopathologically normal appendix with nonsurgical disease was 5.08 days. The mean hospital stay in patients with surgical cause other than acute appendicitis was 8.5 days.

Table-2 Score distribution and recommendation in patients with positive and negative appendicectomies.

\begin{tabular}{|l|l|l|l|l|}
\hline Score & $\begin{array}{l}\text { Positive } \\
\text { appendicectomy } \\
\text { number (\%) }\end{array}$ & $\begin{array}{l}\text { Negative appendicectomy } \\
\text { number (\%) }\end{array}$ & $\begin{array}{l}\text { Total number } \\
(\%)\end{array}$ & Recommendation \\
\hline$<8$ & $33(5.54)$ & $29(33.33)$ & $62(9.07)$ & Do not operate \\
\hline $8>12$ & $96(16.11)$ & $35(40.23)$ & $131(19.18)$ & $\begin{array}{l}\text { Wait and } \\
\text { Reevaluate }\end{array}$ \\
\hline$>12$ & $467(78.36)$ & $23(26.44)$ & $490(71.74)$ & Operate \\
\hline Total & $596(87.26)$ & $87(12.74)$ & $683(100)$ & \\
\hline
\end{tabular}

It is evident from table- 2 that , $71.74 \%$ patients had score of more than 12 which suggests need for operation , 467 out of them had positive appendicectomy and 23 out of them had histopathologically normal appendix. One of them had right tubo ovarian mass and the rest 22 had no detectable pathology. These patients were relieved of their pain post operatively and had no complaints till last follow up. 131 patients had scores between 8 to 12 which was in the equivocal range and suggests observation and reevaluation. 96 out of 131 have positive appendectomies. 62 patients score less than 8 which was against the diagnosis of acute appendicitis but 33 of them had histologically proved positive appendicectomy. Only $5.54 \%$ of positive appendicectomies was in the score category of less than 8 while $78.36 \%$ of positive appendicectomies fall in the score category of more than 12 . Whereas, $33.33 \%$ of negative 
appendicectomies was in the score category of less than 8 and only $26.44 \%$ of negative appendicectomies was in the more than 12 score category.

\section{$\%$ of negative appendicectomy}

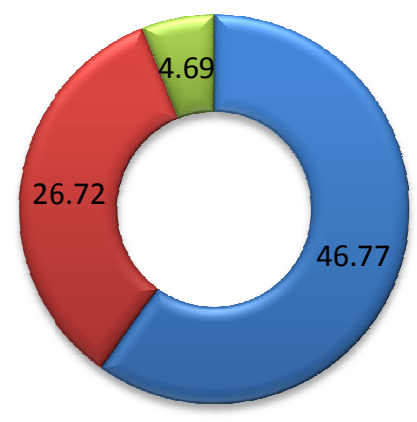

$\square<8$

$\nabla 812$

$\square>12$

Fig-1 Doughnut showing percentage of negative appendicectomies in the three score groups.

Thus, if the present scoring system is followed the negative appendicectomy rate would be $4.69 \%$ (fig-1).

The number of false negative results in female $(11.11 \%)$ was less than that in male $(29.55 \%)$. Thus, it appears that the present scoring system is more sensitive for female and the difference is statistically significant. The overall false negative result was $22.31 \%$.

Table-3 Showing histopathological diagnosis in patients with score $>12$.

\begin{tabular}{|l|l|l|l|}
\hline \multirow{2}{*}{ Sex } & Score $>12$ & Total Number \\
\cline { 2 - 4 } & $\begin{array}{l}\text { Positive Negative } \\
\text { Appendicectomy number (\%) number (\%) }\end{array}$ & \\
\hline Male & $248(53.10 \%)$ & $18(78.26 \%)$ & $266(54.29 \%)$ \\
\hline Female & $219(46.90 \%)$ & $05(21.74 \%)$ & $224(45.71 \%)$ \\
\hline Total & $467(78.36 \%)$ & $23(26.44)$ & $490(100 \%)$ \\
\hline
\end{tabular}

$\mathrm{P}$ value $=<0.05$

Table no -3 shows that score was more than 12 in 266 male patients and 248 of them have histologically proved positive appendicectomy.224 female had score more than 12 , out of them 219 have positive appendicectomy. The number of false positive results on using the present scoring system was $26 \%$ (23 out of 490 ). It is $2 \%$ (5 out of 224 ) in female and $6 \%$ (18 out of 266) in males. So it appears that the score is more specific for female and the difference is statistically significant. In the present study, wound infection was the only postoperative complication . 27 patients had postoperative wound infection out of which 26 were from positive appendicectomy group and one from negative appendectomy group.

DISCUSSION: The diagnosis of acute appendicitis continues to be difficult due to variable presentation of the disease and the lack of reliable diagnostic test. Although there has been some improvement in the diagnosis of acute appendicitis over the past decades, the percentage 
of normal appendix reported in various series varies from 8-33\% [10, 11,12,32]. Delayed diagnosis and negative appendectomies both causes significant morbidity and mortality. Negative exploration is not innocuous and carries a morbidity that has been estimated as high as $15 \%$ [13]. Decision making in cases of acute appendicitis poses a clinical challenge especially in developing countries where advanced radiological investigations do not appear cost effective and so clinical parameters remain the mainstay of diagnosis. Hence we have developed our practical scoring system to diagnose acute appendicitis preoperatively.

In the present study 683 patients who were operated for acute appendicitis with preoperative diagnosis of acute appendicitis were studied. The maximum incidence of positive appendicitis (88.59\%) was in the age group of $11-30$ years which is comparable with the previous studies of the different authors [1,2,3].The higher incidence of positive appendicectomy in this age group can be explained on the basis of lymphoid hyperplasia is at peak at this age. Male to female ratio in patients with positive appendicectomy is $1.5: 1$ which is comparable to the other series $[5,11,14,15,16]$. The incidence of negative appendectomies in the present study is $12.74 \%$.Similar results were reported in literature: $21 \%[3], 7 \%[6]$, 6.84\%[11],13\% [18], 15.6[19].

There are even opinions and evidences that if negative appendicectomy rates are below $10-15 \%$, the surgeon is operating on too few patients thus increasing the risk of complications $[7,13,15,19,20,21,22]$.

The maximum number of negative appendicectomies in the present study was in the females between the age group of 11-30 years( 53 out of 87), which is comparable with the results of [13] who quoted highest incidence of error in 21-40 years of female and error in diagnosis was twice in females than in male. This age group is the peak child bearing age of women and most of the time gynecological disorders are confused with acute appendicitis. A histological normal appendix does not always mean a normal appendix. Some histologically normal appendices in patients with acute pain in right iliac fossa contain abnormal concentration of neuropeptides which may explain the relief of pain after removal of a histologically normal appendix observed by different authors[12,24,25].The abnormal content of neuropeptides and the observed neuronal sprouting possibly combined with the immunological response could be part of the pathogenesis of pain in patients with a clinical diagnosis of acute appendicitis and a histologically normal appendix[24,25].

Majority (90\%) of patients with positive appendicectomy presented within 24 hours while only $10 \%$ of patients with negative appendicectomy presented within 24 hours in present study. Thus, as the duration of pain increases chances of negative appendicectomies increase. Similar results were also seen in the study by [26].

The test performance characteristics of the developed scoring system in the 683 patients studied in our series were derived by using computer software XLSTA-2012 and AUTOCAD-2010.

Table-4 Showing sensitivity and specificity.

\begin{tabular}{|l|l|l|}
\hline Total score cut point & Sensitivity & Specificity \\
\hline$>4$ & 1.00 & 0.00 \\
\hline$>8$ & 0.74 & 0.48 \\
\hline$>12$ & 0.82 & 0.60 \\
\hline
\end{tabular}


Using the above table, ROC curve of the present study is plotted as shown

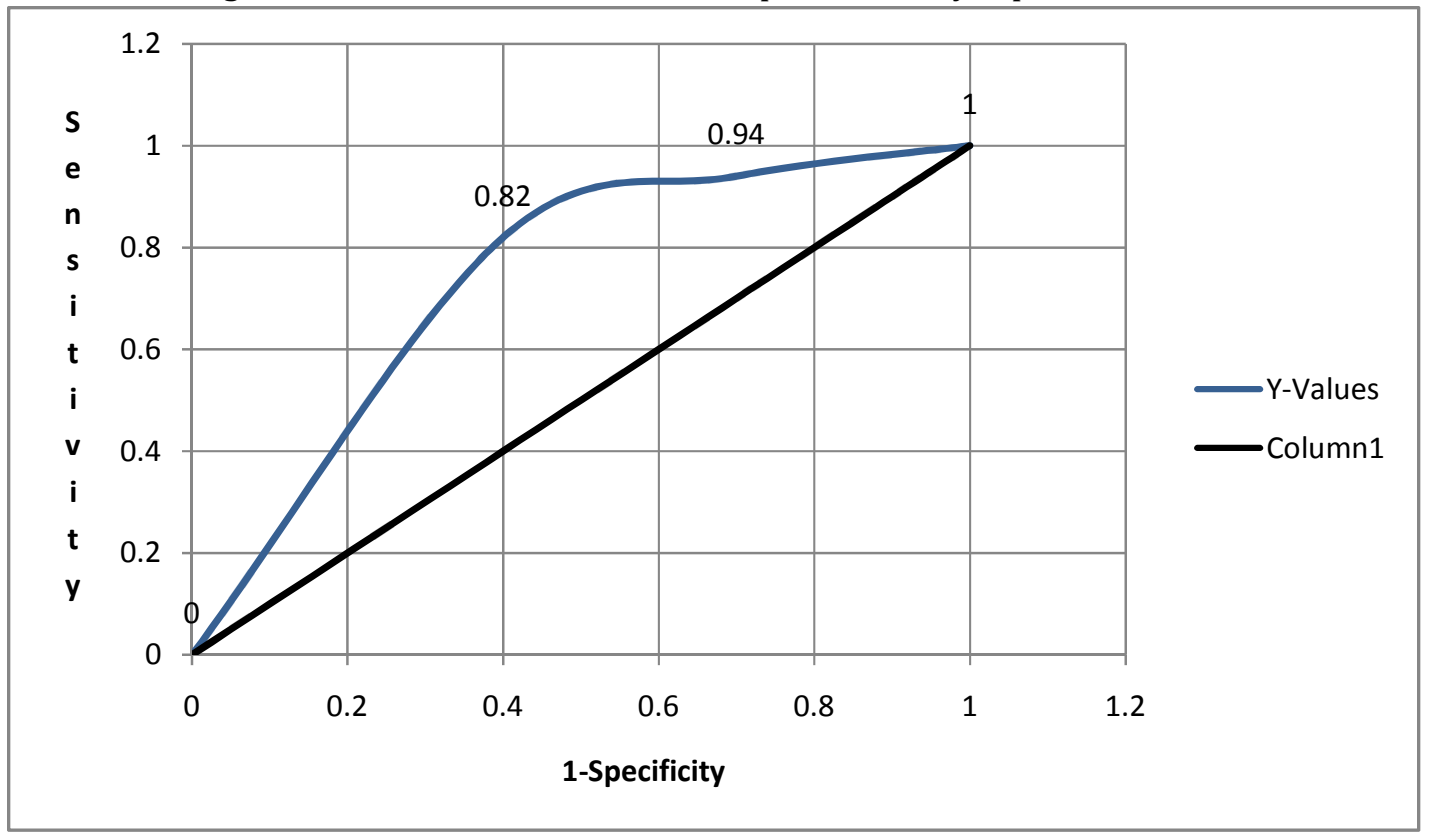

Area under ROC curve $=70.0573 \%$.

ROC curve suggests that the score of 12 is the best cut off that yields maximum sensitivity and specificity. The 0.73 sensitivity and 0.87 specificity was reported by the author [8]. Area under the curve is $70.06 \%$ which suggest a good predictive value of this scoring system.

Score more than 12 is considered as the best cut point for making decision rule was developed from the ROC curve. The score of 12 was found to be the best cut point for making a decision to operate or not in present study. Total score of more than 12 was observed in $490(71.74 \%)$ patients. Out of these, 467 patients had histologically inflamed appendix. 4 patients had histologically normal appendix but the total score was more than 12 . One of these 4 patients had right tubo ovarian mass which can explain the positive clinical signs and symptoms (rebound tenderness in right iliac fossa, PR tenderness on right side) resulting in high total score. In the rest of 3 patients no other pathology was detected. Postoperatively these patients had no complaints. These cases may be a part of the group in which the appendix which explain the relief of pain after removal of the appendix as reported by $[25,26]$.

The scoring system suggested the observation and reevaluation in 131 patients as their total score was between 8 and 12.96 of them had histological evidence of inflamed appendix. 95 out of these 96 patients did not give any history of migration of pain. Migration of pain is a subjective parameter and is given a high score of 6 in our scoring system. This may account for the low initial score in this group of patients. One patient gave positive history of migration of pain but there was no history of nausea or vomiting and no PR tenderness, thus causing the score to go below 12.These patient might have been identified later during the observation and reevaluation period by the development of rebound tenderness, PR tenderness or nausea / vomiting. 35(40.23\%) of the 131 patients had negative appendicectomy. Two of these patients had ruptured ectopic and 3 had Meckel's diverticulitis, thus, explaining the positive clinical findings of rebound tenderness and history of nausea or vomiting causing the score to rise above 8 . These patients had no history of migration of pain. In rest of patients no pathology was 
detected. Rebound tenderness was present in all of them which had a high score of 5 in our scoring system. 3 of them had history of nausea and vomiting. These patients with histological normal appendix might be the patients of neuro immune appendicitis. Migration of pain was absent in all of them which can explain a score of less than 12 in them.

62 Patients had score below 8 and 33 of them had histopathological evidence of inflamed appendix. 32 out of these 33 patients give no history of migration of pain which may account for the low score in these patients. Maximum patient with pulse rate between 92 to 100 per minute had appendicitis. Tachycardia( score of -0.1 in the present study ) i.e. pulse rate above $90 / \mathrm{min}$ was found in $387(64.93 \%$ ) patients with positive appendix while only $35(40.23 \%)$ patients with negative appendicectomies had pulse rate above $90 / \mathrm{min}$. Ganesh Roy (1969) found tachycardia in $67.6 \%$ of patients. Samsi found tachycardia in $62 \%$ of patients. Thus, the presence of tachycardia has been consistently shown to support the diagnosis of acute appendicitis. Rebound tenderness was absent in 6 out of these 33 patients, PR tenderness and history of nausea or vomiting was present in only 2 of them. But all of these 33 patients had tenderness in right iliac fossa. One of them had rebound tenderness and raised leukocyte count in addition to the pain in right iliac fossa, but the total score was only 4.9 due to absence of history of migration of pain and nausea or vomiting, absent PR tenderness and pulse of 98 per minute. Another patients with a score 3.9 had tachycardia and tenderness in right iliac fossa, raised polymorphs count and history of nausea and vomiting present. But the score was low due to the absence of history of migration of pain, rebound tenderness signs were negative and PR tenderness was absent. Thus, in spite of having so many clinical parameters and leukocyte counts in favor of the diagnosis of acute appendicitis, these patients had low score. The fact that none of the classical feature is invariably present in all patients of acute appendicitis is known to us since old times. And so, few false negative results can always be expected in any scoring system. As outlined in the review of literature, the studies evaluating the various scoring systems devised for the diagnosis of acute appendicitis have given differential results and none of the scoring system has proved to be totally dependable in all the trial which were conducted to evaluate its efficacy in the accurate diagnosis of acute appendicitis. But how many patients of acute appendicitis are missed clinically and hence do not undergo appendicectomy is not known. The detection of false negative results of the clinical evaluation will require laparotomy in all the patients with pain in right iliac fossa which is medically, ethically and financially not feasible. It may be much higher than that of the scoring system if one accepts the concept of spontaneous resolution of non obstructive acute appendicitis.

A study to determine the accuracy of a protocol for diagnosis of appendicitis in children based on clinical evaluation by a pediatric surgeon with selective use of diagnostic imaging studies showed that the sensitivity of the protocol was $99 \%$, specificity was $92 \%$, positive predictive value was 95\%, and negative predictive value was 99\%. The accuracy was 97\% compared with an accuracy of $82 \%$ for ultrasound alone and $90 \%$ for CT scan alone. These data show that a protocol based on clinical evaluation by a pediatric surgeon with selective use of imaging was highly accurate for the diagnosis of appendicitis in children. Low rates of negative appendectomy (5\%) and perforation (18\%) were achieved without the potential costs and radiation exposure of excess imaging [1]. Despite the availability of radiological (US/CT) investigative modalities, a recent population -based study in USA indicated that there was essentially no change in the frequency of negative appendicectomy [22]. Similar results were also reported, where the authors found Ultrasonography did not have any additional benefit 
over Alvarado score and were of the opinion that Ultrasonography is unnecessary in diagnosis of acute appendicitis [27].

Various scoring systems are being used to aid the diagnosis of acute appendicitis and bring down the negative appendicectomy rates. An open prospective study to validate simplified scoring system carried out at Sweden. Out of 475 patients, 392 had histological verified appendicitis. The negative laparotomy rate was $17.5 \%(11.2 \%$ for men and $25.4 \%$ for women). The sensitivity of the scoring system for appendicitis at the main cut off point was 0.73 and specificity was $0.87 \mathrm{in}$ the study of [8]. In our study sensitivity is $82.9 \%$; the negative laparotomy rate is $12.74 \%$ which is low as compared to other scoring system. If the present bedside scoring system is followed the negative appendicectomy rate would be $4.69 \%$.

Out of 90 patients, 73 patients underwent surgery and appendicitis was confirmed in 68 cases as reported by the author[11]. They used a modification of Alvarado scoring system which consists of eight variables and would provide an accurate guide to the preoperative diagnosis of acute appendicitis. They were given specific scores according to variables of scoring system and divided into 3 groups. Group III patients (score 7 or more) underwent surgery, group II (score 5-6) were admitted for close observation and group I (score 4 or less) were discharged home. Patients from group II with increased symptom intensity (score 7 or more) on re-evaluation underwent surgery. The negative appendicectomy rate was $6.84 \%$, perforation rate was $36.9 \%$. Positive predictive value was $93.1 \%$ in their series.

A study of surgeon performed ultrasound as a diagnostic tool in appendicitis showed that combining a clinical examination with surgeon performed ultrasound(SPUS) increases diagnostic accuracy (90\%) and may be used as a primary diagnostic tool and computed tomography reserved for challenging cases, limiting costs and radiation exposure[28].

A study to evaluate the utility of C-reactive protein(CRP), Pro calcitonin, D-lactate and WBC count as an aid to distinguish appendicitis from other diagnosis found that WBC count $>12000$ cells/cubic mm (Adjusted odds ratio 6.54) and CRP levels $>3 \mathrm{mg} / \mathrm{dl}$ (Adjusted odds ratio3.44) increases the likelihood of appendicitis[28].In a another study of correlation of clinical data with histopathological findings in childhood appendicitis showed a significant correlation of "leukocyte count", "vomiting" and "percussion tenderness" with histopathology only in $75 \%$ of children and found out even with these statistical parameters a comparably high percentage of false positive appendicectomies has to be anticipated [30].

The complication rate in our study was $4.68 \%$ due to wound infection in 32 patients without any mortality. A negative appendicectomy had a negligible mortality and morbidity of around $10 \%$ was observed by author [27]. Sabiston has quoted complication like wound sepsis in $6.35 \%$ of cases [31].

CONCLUSION: - Thus, the scoring system developed from a local database works well and can aid in reducing the negative appendicectomy rate and in diagnosing acute appendicitis accurately. The prospective evaluation of the present scoring system showed good test performance characteristics, it can be especially useful for residents and junior surgeon with limited clinical experience in accurately diagnosing the patients of acute appendicitis and avoiding negative appendicectomies. 


\section{REFERENCES:}

1. Kosloske AM, Love CL, Rohrer JE, et al (2004) The diagnosis of appendicitis in children: outcomes of a strategy based on pediatric surgical evaluation. Pediatrics. 113 (1 Pt 1):29.

2. Dado G, Anania G, Baccarani U et al (2000) Application of a clinical score for the diagnosis of acute appendicitis in childhood. J.Pediatr Surg 35:1320-1322.

3. Chan MY (2003) Alvarado score as admission criteria in patients with right iliac fossa pain. Surg.J.Coll.Surg.Edinb.1:39-41.

4. Ohmann C, Franke C, Yang Q (1999) clinical benefit of a diagnostic score for appendicitis. Results of prospective international study. Archives of surgery.134 (9):993-6.

5. Kalan M, Rich AJ, Talbol D (1994) Evaluation of the modified Alvarado score in the diagnosis of acute appendicitis. A prospective study. Ann. R. Coll.Surg.Engl.76:418-419.

6. Jawaid A, Asad A, Motiel A et al(1999) Clinical scoring system; a valuable tool for decision making in cases of acute appendicitis. J. Pak Med Assoc.49 (10):254-9.

7. Ohmann C, Yang Q, Franke C (1995) Diagnostic scores for acute appendicitis. Adominal pain study group. European J.of surgery; 161(4):273-81.

8. Fenyo G, LindbergG, Blind $P$ et al (1997) Diagnostic decision support in suspected acute appendicitis, Vallidation of a simplified scoring system.Eur J Surg.163:831-38.

9. Hussain A, Mahmood H, Singhal T (2009) What is positive appendicitis? A new answer to an old question. Clinical, macroscopical and microscopical findings in 200 consecutive appendectomies Singapore Med J. 50(12):1145-9.

10. Phillips AW, Jones AE, Sargen K. (2009) Should the macroscopically normal appendix be removed during laparoscopy for acute right iliac fossa pain when no other explanatory pathology is found? Surg Laparosc Endosc Percutan Tech. 19(5):392-4.

11. Shera AH, Nizami FA, Malik AA et al (2011) Clinical scoring system for diagnosis of acute appendicitis in children.Indian J Pediatr.78(3) : 287-90.

12. Deutsh A, Shani N, Reiss R (1983) are some appendectomies unnecessary. An analysis of 319 white appendices.J.R.Coll.Surg.Edinb.28:35-40.

13. Anderson RE, Hugander A (1992) Diagnostic accuracy and perforation rate in appendicitis association with age and sex of patients and appendicitis rate. Eur.J. Surg.158:37-41.

14. Groonoos JM, Groonoos P (1999) Leucocyte count and C-reactive protein in the diagnosis of acute appendicitis. B.J.Surg:86:501-504.

15. Naqi A (2001) Role of Alvarado scores in diagnosis of acute appendicitis. Pakistan J.Surgery. 17(3):41-46.

16. Bhattacharjee PK, Chowdhary T, Roy D (2002) Prospective evaluation of modified Alvarado score for diagnosis of acute appendicitis. JIMA may 2002.

17. Dey S, Mohanta PK, Barush AK (2010) Alvarado scoring in acute appendicitis - a clinicopathological correlation. Indian J. Surg. 72(4):290-293.

18. Khan I, Rehman A (2005) Application of Alvarado score in diagnosis of acute appendicitis. J Ayub Med Coll Abbottad 17(3):41-44.

19. Denizbasi A, Unluer EE (2003) the role of the emergency medicine resident using the Alvarado scores in the diagnosis of acute appendicitis compared with the general surgery resident. Eur J Emerg Med 10(4):296-301. 
20. Erikson S,Granstrom L,Caristrom A(1994) The diagnostic value of repetitive preoperative analysis of C-reactive protein and total leukocyte count in patient with suspected acute appendicitis. Scand J Gastroenterol.29 (12):1145-9.

21. Gurlayik E, Unalmiser S et al (1995) Accuracy of serum C - reactive protein measurement in diagnosis of acute appendicitis compared with surgeons clinical impression. Dis Colon Rectum.38 (12):1270-74.

22. Abdeldaim Y, Mahmood S, Mc Avinchey D (2007) The Alvarado score as a tool for diagnosis of acute appendicitis. Ir. Med J.100 (1):342.

23. Disebastiano P, Fink T, Dimola FF et al (1999) Neuroimmune appendicitis. Lancet; 354:461-466.

24. Wang Y, ReenDJ, Puri P (1996) is a histologically normal appendix following emergency appendectomy always normal? Lancet.347:1076-1079.

25. Stead RH, Franks AJ, Goldsmith (1990) Mast cells, nerves and fibrosis in the appendix: a morphological assessment. J.Pathol; 161:209-19.

26. Pieper R, Kager L, Nasman P (1982) acute appendicitis a clinical study of 1018 cases of emergency appendectomy.Acta Chir Scand.148:51-62.

27. Baidya N, Rdrigues G, Rao A, et al (2007) Evalution of Alvarado score in acute appendicitis: a prospective study. Internet j. surg 9(1).

28. Burford JM, Dassinger MS (2011) Surgeon performed ultrasound as a diagnostic tool in appendicitis Smith Journal of pediatric Surgery. 46(6): 1115-1120.

29. Kwan KY., Nager AL (2010) Diagnosing pediatric appendicitis: The American Journal of Emergency Medicine. 28(9): 1009-1015.

30. Müller AM, Kaucevic M, Coerdt W (2010] Appendicitis in childhood: correlation of clinical data with histopathological findings Klin Padiatr. 222(7):449-54.

31. Kevin P,L, Charles SC, Richard JA(2001) Appendix.ch.45.Text book of surgery, Sabiston $16^{\text {th }}$ edition WB Saunders company.917-927.

32. Tamanna MZ,Eram U, Hussain AM et al (2012) Alvarado score in diagnosis of acute appendicitis.International J.of Basic and applied medical sciences.2(1):66-70. 